\title{
3D cone-beam CT with a twin robotic $x$-ray system in elbow imaging: comparison of image quality to high-resolution multidetector $\mathrm{CT}$
}

Jan-Peter Grunz ${ }^{1 *}$ DD, Andreas Max Weng ${ }^{1}$, Andreas Steven Kunz ${ }^{1}$, Maike Veyhl-Wichmann², Rainer Schmitt ${ }^{1}$, Carsten Herbert Gietzen ${ }^{1}$, Lenhard Pennig ${ }^{3}$, Stefan Herz ${ }^{1}$, Süleyman Ergün² ${ }^{2}$ Thorsten Alexander Bley ${ }^{1}$ and Tobias Gassenmaier ${ }^{1}$

\begin{abstract}
Background: Elbow imaging is challenging with conventional multidetector computed tomography (MDCT), while cone-beam $C T(C B C T)$ provides superior options. We compared intra-individually $C B C T$ versus MDCT image quality in cadaveric elbows.

Methods: A twin robotic x-ray system with new CBCT mode and a high-resolution clinical MDCT were compared in 16 cadaveric elbows. Both systems were operated with a dedicated low-dose (LD) protocol (equivalent volume $\mathrm{CT}$ dose index $\left.\left[\mathrm{CTDI}_{\text {vol }(16 \mathrm{~cm})}\right]=3.3 \mathrm{mGy}\right)$ and a regular clinical scan dose $(\mathrm{RD})$ protocol $\left(\mathrm{CTDI}_{\mathrm{vol}(16 \mathrm{~cm})}=13.8 \mathrm{mGy}\right)$. Image quality was evaluated by two radiologists (R1 and R2) on a seven-point Likert scale, and estimation of signal intensity in cancellous bone was conducted. Wilcoxon signed-rank tests and intraclass correlation coefficient (ICC) statistics were used.

Results: The CBCT prototype provided superior subjective image quality compared to MDCT scans (for RD, $p \leq 0.004$; for $L D, p \leq 0.001$ ). Image quality was rated very good or excellent in $100 \%$ of the cases by both readers for RD CBCT, $100 \%$ (R1) and 93.8\% (R2) for LD CBCT, $62.6 \%$ and $43.8 \%$ for RD MDCT, and $0.0 \%$ and $0.0 \%$ for LD MDCT. Single-measure ICC was 0.95 (95\% confidence interval 0.91-0.97; $p<0.001$ ). Software-based assessment supported subjective findings with less "undecided" pixels in CBCT than dose-equivalent MDCT $(p<0.001)$. No significant difference was found between LD CBCT and RD MDCT.
\end{abstract}

Conclusions: In cadaveric elbow studies, the tested cone-beam $C T$ prototype delivered superior image quality compared to high-end multidetector $C T$ and showed a potential for considerable dose reduction.

Keywords: Cancellous bone, Cone-beam computed tomography, Elbow, Elbow joint, Multidetector computed tomography

\footnotetext{
* Correspondence: Grunz_J@ukw.de

${ }^{1}$ Department of Diagnostic and Interventional Radiology, University Hospital

Würzburg, Oberdürrbacher Straße 6, 97080 Würzburg, Germany

Full list of author information is available at the end of the article
}

\section{Springer Open}

(c) The Author(s). 2020 Open Access This article is licensed under a Creative Commons Attribution 4.0 International License, which permits use, sharing, adaptation, distribution and reproduction in any medium or format, as long as you give appropriate credit to the original author(s) and the source, provide a link to the Creative Commons licence, and indicate if changes were made. The images or other third party material in this article are included in the article's Creative Commons licence, unless indicated otherwise in a credit line to the material. If material is not included in the article's Creative Commons licence and your intended use is not permitted by statutory regulation or exceeds the permitted use, you will need to obtain permission directly from the copyright holder. To view a copy of this licence, visit http://creativecommons.org/licenses/by/4.0/. 


\section{Key points}

- The cone-beam computed tomography (CT) prototype provides better subjective image quality than dose-equivalent high-end multidetector $\mathrm{CT}$ in cadaveric elbow scans.

- Software-assisted differentiation between the cancellous bone and fatty marrow was superior using the new cone-beam CT scanning mode.

- Considerable dose reduction over multidetector CT is possible without loss of image quality.

\section{Background}

Cone-beam computed tomography (CBCT) has been an integral part of dental imaging for many years $[1,2]$. During the last decade, it has also made its way into musculoskeletal imaging [3].

With the excellent depiction of the osseous tissue and the establishment of dedicated extremity scanners, CBCT provides several advantages over conventional multidetector CT (MDCT), particularly for trauma imaging [4]. Current literature on CBCT is mostly focused on lower extremity and spine imaging under weight-bearing conditions [5-7]; however, upper extremity imaging benefits from $\mathrm{CBCT}$ as well.

While the upsides of the cone-beam technology have been examined for wrist and finger imaging before [810], the application to the elbow region has not been well established in the literature so far. Dislocations and fractures involving the elbow joint are common findings after trauma, especially in children [11]. The diagnostic routine usually comprises plain radiography for fracture diagnosis, although severely displaced fracture patterns or suspected occult coronoid or radial head fractures may require additional MDCT imaging for further evaluation and surgical planning [12].

Comparable to wrist scans, elbow imaging in MDCT is mostly performed in a modified so-called "superman" position to assure the best possible image quality and lowest radiation dose [13]. Yet, empirical knowledge shows that patients with elbow injuries or impaired shoulder movement and older patients in general are oftentimes unable to adopt or maintain that posture. Extremity-dedicated CBCT scanners provide more comfortable positioning options, allowing for optimal imaging conditions even if the patient has physical disabilities or is in pain [14]. However, due to technical limitations such as small field of view, many CBCT scanners can only be used for a limited number of imaging tasks, lacking the versatility of a conventional MDCT scanner [3].

The multifunctional $x$-ray device we employed in the study herein presented provides more flexibility. Its two telescopic arms and large detector allow for three- dimensional examination of most body parts and additional radiography and fluoroscopy. In earlier studies with a commercially available CBCT scan mode, a considerable reduction of radiation dose over standard MDCT was achieved for ankle [15] and wrist [16] studies using automatic tube current modulation. In this work, our goal was to establish reproducible clinical scan protocols with fixed acquisition parameters, thus enhancing comparability between the new CBCT prototype for the twin-robotic $\mathrm{x}$-ray system and high-end MDCT in cadaveric elbow scans.

\section{Methods}

\section{Cadaveric specimens}

We examined the elbow joints of eight, randomly chosen, formalin-fixed cadaveric specimens with the novel prototype cone-beam CT scan mode of a multiuse, twin robotic x-ray system (Multitom Rax, Siemens Healthineers, Erlangen, Germany) and a high-resolution multidetector CT scanner (Somatom Force, Siemens Healthineers, Erlangen, Germany). MDCT scans were conducted in a modified "superman" stance, while CBCT studies were performed using the tableside scan position for 3D imaging of the upper extremity. The scan postures for MDCT and CBCT elbow imaging are presented by a staff member in Fig. 1.

\section{Technical parameters and dose assessment}

The $\mathrm{x}$-ray system tube and flat panel detector are mounted on two motor-driven, telescopic arms that are connected to ceiling rails. While independent arm movement facilitates two-dimensional and fluoroscopy imaging, both arms move concurrently along predetermined paths for the acquisition of 3D projection data in CBCT scan mode (Fig. 2). The input field of the flat panel detector measures $22.8 \mathrm{~cm} \times 21.3 \mathrm{~cm}$ with a 3D image matrix of $1,540 \times 1,440$ pixels (pixel size $148 \mu \mathrm{m}$ ). The $\mathrm{x}$-ray tube is capable of voltages from 40 to $150 \mathrm{kV}$ and currents from 0.5 to $800 \mathrm{mAs}$.

The tableside trajectory used for elbow and wrist imaging has a sweep angle of $200^{\circ}$ and an asymmetric source-to-image-distance of $115 \mathrm{~cm}$. Due to an increase in maximum frames per second from 16 to 30 , the total scan time is reduced with the new prototype from 20 to $12 \mathrm{~s}$ when compared to the commercially available software version. Taking into account the two telescopic arms acceleration and deceleration phase, 304 projection images are acquired during each scan. Dose levels and subsequently tube current can be varied for different scan protocols with an automatic dose modulation system keeping the detector dose level constant. Therefore, a sensor at the detector continuously measures the incoming radiation and the automatic 

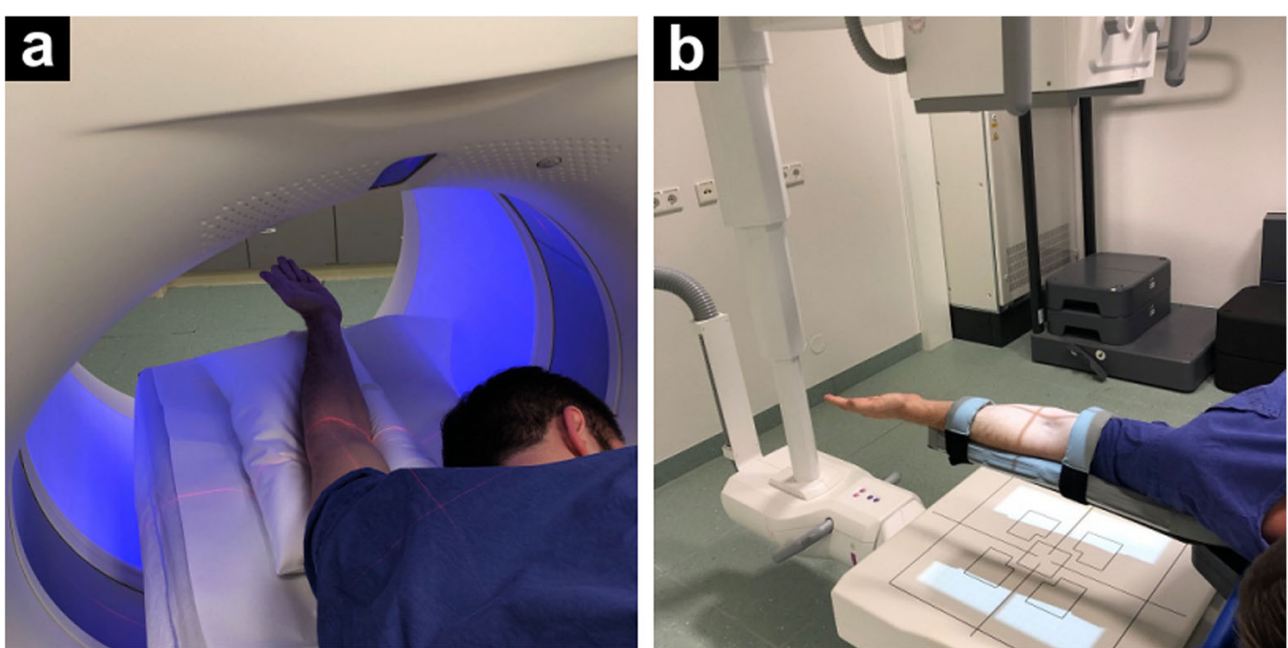

Fig. 1 Positioning for image acquisition using a contemporary high-end multidetector computed tomography scanner (a) according to the modified "superman" position, and the twin robotic x-ray system cone-beam computed tomography scan mode (b) with the elbow positioned besides the patient table

exposure control modifies the tube current-time product accordingly to the preset dose values.

For this study, however, we decided to use constant tube currents to provide comparable circumstances for both imaging modalities. To compare the applied radiation dose between $\mathrm{CBCT}$ and $\mathrm{MDCT}$, the dosearea product of the CBCT scan mode was multiplied by a linear scaling factor leading to volume computed tomography dose index $\left(\mathrm{CTDI}_{\mathrm{vol}}\right)$-equivalent values. By using a polymethyl methacrylate dosimetry phantom, conforming to International Electrotechnical Commission 60601-2-44:2009 with a total length of $300 \mathrm{~mm}$ and a diameter of $160 \mathrm{~mm}$ as well as a

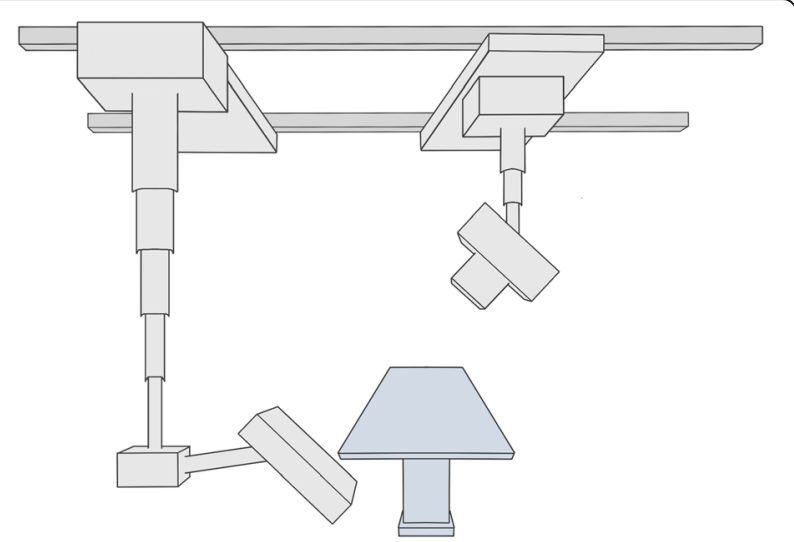

Fig. 2 The prototype cone-beam computed tomography scan mode is based on a radiography system with two telescopic arms mounted on ceiling rails. One arm carries the $x$-ray tube, while the other holds a large flat panel detector. Simultaneous arm movement along predefined trajectories allows for three-dimensional projection data acquisition standard dosimetry system (Nomex Dosimeter, PTW, Freiburg, Germany) with a $300 \mathrm{~mm}$ ionisation chamber, the scaling factor for each combination of voltage, pre-filtration and acquisition geometry was calculated in advance. To achieve this, the doselength product (DLP) was quantified in five chambers. Then, the standard weighting scheme for dose measurements was applied to each value to determine the DLP $_{\text {vol }}$ values. CTDI ${ }_{\text {vol }}$ values were calculated by dividing the DLP $_{\mathrm{vol}}$ by the beam width (equivalent to the field of view in the $z$-direction), and to finally compute the scaling factor, $\mathrm{CTDI}_{\mathrm{vol}}$ was divided by dose-area product values. We operated with a dedicated low-dose

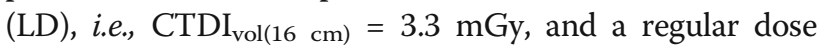
protocol (RD) comparable to our clinical scan protocol, i.e., $\left.\mathrm{CTDI}_{\mathrm{vol}(16 \mathrm{~cm})}=13.8 \mathrm{mGy}\right)$, for both systems. $\mathrm{MDCT}$ images were acquired using the CT scanner in single-energy mode. Scan parameters for CBCT and MDCT studies are displayed in Table 1.

\section{Image reconstruction parameters}

For the multidetector CT system, scanner-side reconstruction of raw data was conducted using a dedicated bone kernel (Ur77; Siemens Healthineers, Erlangen, Germany). The CBCT data was reconstructed with a comparable prototype kernel that provides equivalent standardised resolution numbers in the axial plane according to vendor information. Multiplanar reconstructions (MPR) of the cadaveric elbow scans were then performed for CBCT and MDCT using special software (Syngo via, Siemens Healthineers, Erlangen, Germany). Irrespective of dose protocol or scanner, slice thickness of $1 \mathrm{~mm}$, increment of $0.5 \mathrm{~mm}$, image matrix of $1,024 \times 1,024$ pixels and field of view of $80 \mathrm{~mm}$ were chosen for axial, coronal and sagittal 
Table 1 Scan parameters

\begin{tabular}{|c|c|c|}
\hline Somatom Force $^{1}$ & regular-dose MDCT & low-dose MDCT \\
\hline Reference kVp & 120 & 120 \\
\hline Reference mAs & 100 & 25 \\
\hline $\mathrm{CTDI}_{\mathrm{vol}(16 \mathrm{~cm})}(\mathrm{mGy})$ & 13.8 & 3.3 \\
\hline Scan duration (s) & 1 & 1 \\
\hline Collimation (mm) & $2 \times 32 \times 0.3$ & $2 \times 32 \times 0.3$ \\
\hline Pitch (mm) & 0.8 & 0.8 \\
\hline Multitom Rax & regular-dose CBCT & low-dose CBCT \\
\hline Reference kVp & 80 & 80 \\
\hline Total scan mAs & 757 & 210 \\
\hline $\mathrm{CTDI}_{\mathrm{vol}(16 \mathrm{~cm})}(\mathrm{mGy})$ & 13.8 & 3.3 \\
\hline Scan duration (s) & 12 & 12 \\
\hline Max. frames per second & 30 & 30 \\
\hline Projections per scan & 304 & 304 \\
\hline
\end{tabular}

CTDI $_{\text {vol }(16 \mathrm{~cm})}=$ volume computed tomography dose index $($ for $16 \mathrm{~cm}$ diameter PMMA dosimetry phantom)

${ }^{1}$ (Siemens Healthineers; Erlangen, Germany)

Acquisition parameters for the regular-dose and dedicated low-dose scan protocols in prototype cone-beam $\mathrm{CT}$ (CBCT) and high-end multidetector $\mathrm{CT}$ (MDCT)

planes. We decided on preset values for window width and level of 3,000 and 1,000 Hounsfield units for optimal bone visualisation. However, readers were allowed to change window settings as needed.

\section{Subjective image analysis}

Two independent radiologists with nine (T.G.; Reader 1, R1) and seven (S.H.; Reader 2, R2) years of experience in musculoskeletal radiology evaluated all elbow studies using the Merlin picture archiving and communication system (Phönix-PACS, Freiburg, Germany). In the first step of their reads, observers reviewed all of the images for each MPR in randomised and blinded order. After blinded review of all data was complete, readers were asked to assess whether the presented image quality of each study was sufficient for diagnostic use and to rate the overall image quality on a seven-point Likert scale $(7=$ excellent; 6 = very good; 5 = good; $4=$ satisfactory; 3 = fair; 2 = poor; 1 = very poor). Furthermore, artefacts and image noise in osseous and soft tissue were judged separately using a five-point Likert scale $(5=$ minimal artefacts or noise; $4=$ little artefacts or noise; $3=$ moderate artefacts or noise; 2 = considerable artefacts or noise; $1=$ strong artefacts or noise).

\section{Objective image analysis}

Software-based estimation of the signal intensity distribution in osseous tissue was performed in order to objectify the task of image quality evaluation. First, the range of signal intensities was calculated for image parts containing bone tissue. Subsequently, the fraction of pixels with a signal intensity between 25 and $75 \%$ of the calculated range was computed. Assuming that pixels above the $75 \%$-border equal trabeculae or cortical bone and pixels below the $25 \%$-border represent fatty marrow, the pixels between the borders were deemed as "undecided", representing a mixture of bone and marrow and partly arising from blurred edges between both. Consequently, a larger share of these pixels indicated impaired image quality. We used special numerical computing software (MATLAB Version 2019a, MathWorks, Natick, MA, USA) for objective evaluation of image quality.

\section{Statistical analysis}

Dedicated software (SPSS Statistics, Version 23.0 for Mac, IBM, Amonk, NY, USA) was used to carry out statistical analyses. We present categorical variables (e.g., scale results) as frequencies, percentages and medians, whereas normally distributed data is presented as means \pm standard deviation. The normal distribution of continuous variables was assessed using KolmogorovSmirnov tests. Paired nonparametric variables were compared using Wilcoxon signed-rank tests. For quantification of interrater reliability, we report the intraclass correlation coefficient (ICC) based on the absolute agreement in a two-way random-effects model. The interpretation of ICC values was conducted according to Koo and Li [17] $(<0.50=$ poor reliability; $0.50-0.75=$ moderate reliability; $0.75-0.90=$ good reliability; $>0.90=$ excellent reliability). The $p$ values $\leq 0.05$ were considered to indicate statistical significance.

\section{Results}

\section{Subjective image quality}

Of 64 elbow studies, both readers considered all $16 \mathrm{RD}$ CBCT, 16 LD CBCT, and 16 RD MDCT scans to be suitable for clinical evaluation. For LD MDCT examinations, R1 deemed $37.5 \%$ of studies and R2 $31.3 \%$ of studies insufficient for diagnostic use (score 1 or 2). In contrast, R1 and R2 found the overall image quality to be very good or excellent (score 6 or 7) in 100\% and $100 \%$ of RD CBCT studies, in $100 \%$ and $93.8 \%$ of LD CBCT studies, in $62.5 \%$ and $43.8 \%$ of RD MDCT studies, and in $0.0 \%$ and $0.0 \%$ of LD MDCT studies, respectively. Median Likert scores for R1 and R2 were 7.0 and 7.0 for RD CBCT studies, 6.0 and 6.5 for LD CBCT studies, 6.0 and 5.0 for RD MDCT studies and 3.5 and 3.0 for LD MDCT studies. Significantly superior image quality of CBCT studies in comparison to dose-equivalent MDCT studies was assessed by both readers: RD protocols, $p \leq 0.002$; LD protocols, $p \leq 0.001$. Low-dose CBCT 

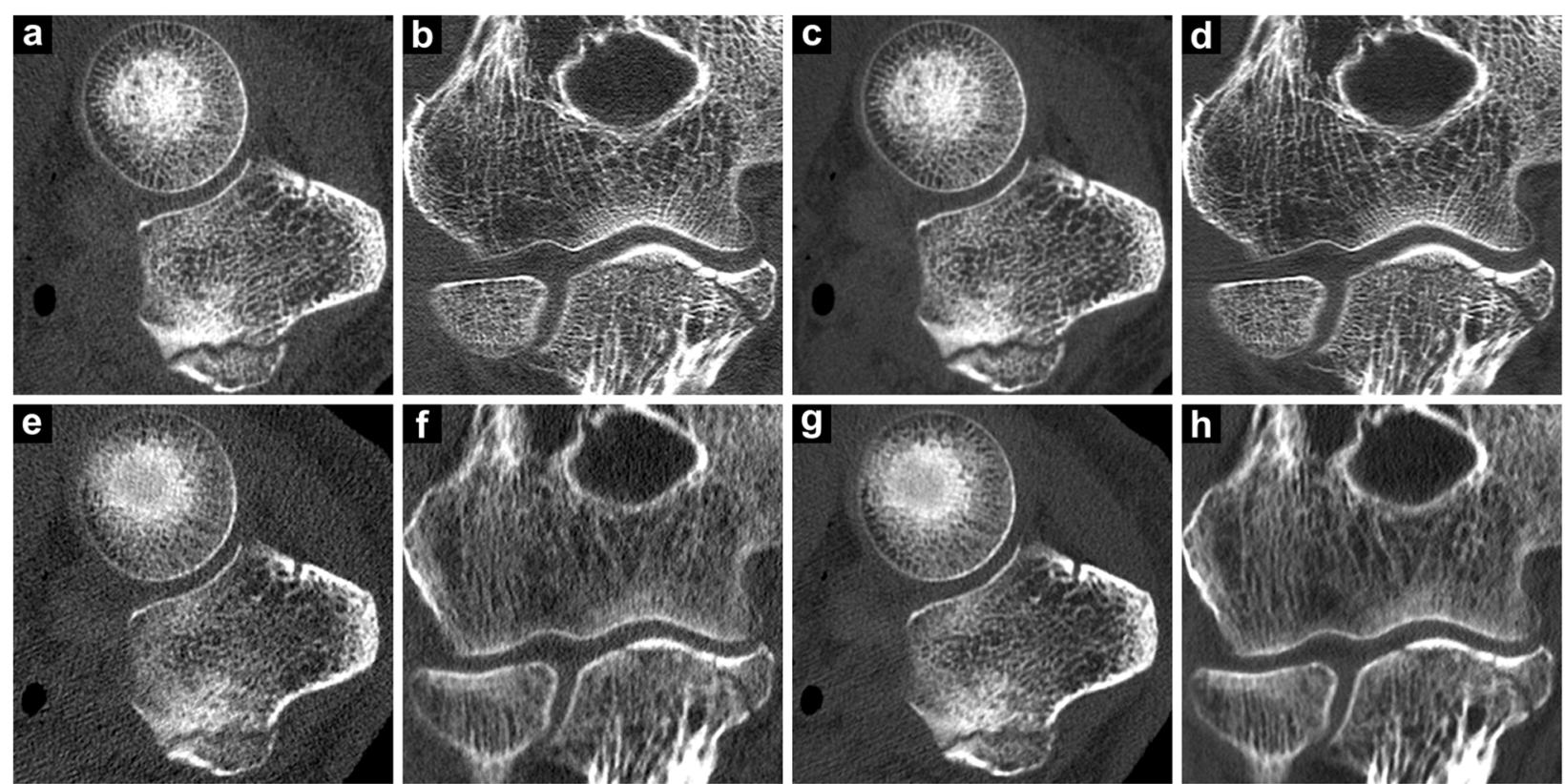

Fig. 3 Representative cone-beam computed tomography (CBCT) and multidetector computed tomography (MDCT) images in the axial and coronal plane with equivalent low-dose and regular-dose scan protocols for visualisation of image quality in cadaveric elbow scans. Images depict an olecranon fracture as an incidental finding. $\mathbf{a}$, b Low-dose CBCT. $\mathbf{c}$, d Regular-dose CBCT. e, f Low-dose MDCT. g, h Regular-dose MDCT

studies were also rated preferably over RD MDCT by both readers $(p \leq 0.004)$. Figure 3 depicts representative coronal and axial MPRs of CBCT and MDCT scans for illustration of overall image quality. Singlemeasure ICC for overall image quality was 0.95 (95\% confidence interval 0.91-0.97, $p<0.001$ ). Table 2 shows detailed results of subjective image quality ratings by both radiologists.

Both observers rated image noise in the bone as less prominent in CBCT than MDCT scans (RD, $p \leq 0.023$; $\mathrm{LD}, p \leq 0.007)$. Noise in the soft tissue was unanimously conceived minor in CBCT for the LD protocol $(p \leq 0.006)$, while R2 also found less soft tissue noise for the RD CBCT protocol $(p=0.019)$. For artefacts in osseous tissue, no difference was assessed between $\mathrm{CBCT}$ and MDCT. In the soft tissue, artefacts were continuously more distinct in CBCT than MDCT scans for both readers (RD, $p \leq 0.003$; LD, $p \leq 0.002$ ) (Table 3).

When comparing both CBCT scan protocols for the elbow imaging on the twin robotic $x$-ray system, both readers conceived $\mathrm{RD} C B C \mathrm{C}$ to be superior to $\mathrm{LD} C \mathrm{CBCT}$

Table 2 Subjective evaluation of cone-beam (CBCT) and multidetector CT (MDCT) image quality by two radiologists (R1, R2) using a seven-point Likert scale

\begin{tabular}{|c|c|c|c|c|c|c|c|c|c|}
\hline & \multirow[t]{2}{*}{ Score } & \multicolumn{2}{|c|}{ Regular-dose CBCT } & \multicolumn{2}{|c|}{ Low-dose CBCT } & \multicolumn{2}{|c|}{ Regular-dose MDCT } & \multicolumn{2}{|c|}{ Low-dose MDCT } \\
\hline & & R1 & R2 & R1 & R2 & R1 & R2 & $\overline{\mathrm{R} 1}$ & R2 \\
\hline \multirow[t]{8}{*}{ Overall image quality } & 7 & $16(100.0)$ & $13(81.3)$ & $7(43.8)$ & $8(50.0)$ & $1(6.3)$ & $3(18.8)$ & - & - \\
\hline & 6 & - & $3(18.8)$ & $9(56.3)$ & $7(43.8)$ & $9(56.3)$ & $4(25.0)$ & - & - \\
\hline & 5 & - & - & - & $1(6.3)$ & - & $3(18.8)$ & $2(12.5)$ & $5(31.3)$ \\
\hline & 4 & - & - & - & - & $4(25.0)$ & $3(18.8)$ & $6(37.5)$ & $2(12.5)$ \\
\hline & 3 & - & - & - & - & $2(12.5)$ & $3(18.8)$ & $2(12.5)$ & $4(25.0)$ \\
\hline & 2 & - & - & - & - & - & - & $5(31.3)$ & $3(18.8)$ \\
\hline & 1 & - & - & - & - & - & - & $1(6.3)$ & $2(12.5)$ \\
\hline & Median & 7.0 & 7.0 & 6.0 & 6.5 & 6.0 & 5.0 & 3.5 & 3.0 \\
\hline
\end{tabular}

Image quality ordinal score: $7=$ excellent; 6 = very good; $5=$ good; $4=$ satisfactory; $3=$ fair; $2=$ poor; $1=$ very poor. Scale results are displayed as frequencies (percentages) and median values 
Table 3 Assessment of image noise and artefacts in the bone and soft tissue for cone-beam (CBCT) and multidetector CT (MDCT) by two radiologists $(\mathrm{R} 1, \mathrm{R} 2)$ using a five-point Likert scale

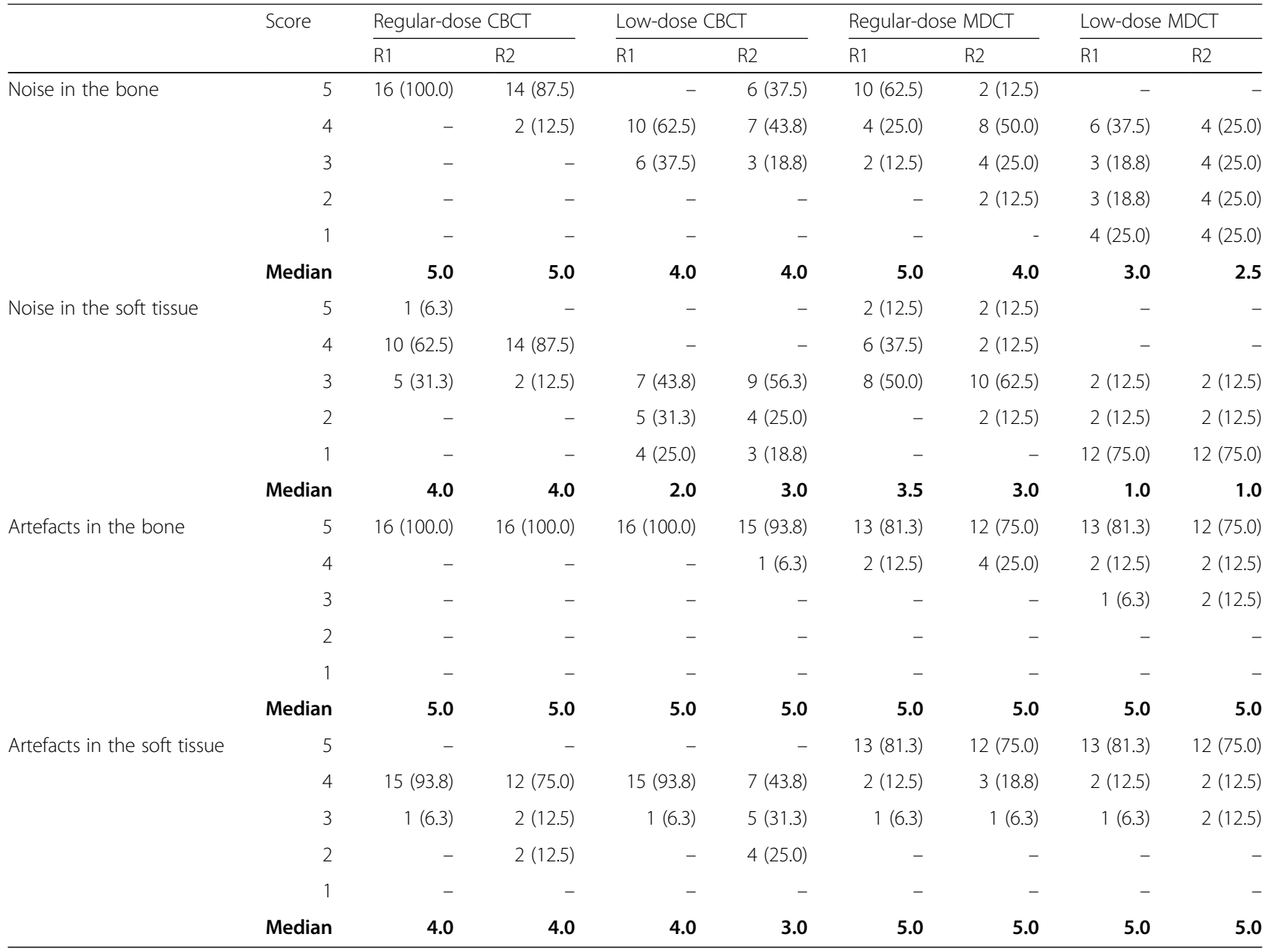

Image quality ordinal score: $5=$ minimal artefacts or noise; $4=$ little artefacts or noise; $3=$ moderate artefacts or noise; $2=$ considerable artefacts or noise; $1=$ strong artefacts or noise). Scale results are displayed as frequencies (percentages) and median values

for image noise in the bone $(p \leq 0.005)$ and soft tissue $(p \leq 0.001)$. Regarding artefacts, R2 found less of them in soft tissue for RD studies $(p=0.008)$, while no significant differences were observed for artefacts in osseous tissue $(p \geq 0.317)$.

\section{Objective image quality}

As displayed in Fig. 4, RD CBCT (median fraction 0.67, IQR 0.16) delivered a smaller fraction of pixels within the $25 \%$ to $75 \%$ range of signal intensity than LD CBCT (0.79, IQR 0.08; $p<0.001)$, RD MDCT (0.81, IQR 0.17; $p<0.001)$, and LD MDCT scans (0.90, IQR 0.05; $p<0.001)$. While LD CBCT scans provided less "undecided" pixels than dose-equivalent LD MDCT scans $(p<0.001)$, no significant difference was found between LD CBCT and RD MDCT studies $(p=0.513)$. The signal intensity distribution in cancellous bone is visualised in Fig. 5.

\section{Discussion}

We compared the image quality of a multi-use $\mathrm{x}$-ray system prototype CBCT scan mode to a high-end MDCT using $\mathrm{CTDI}_{\mathrm{vol}}$-matched, clinical RD and dedicated LD protocols for cadaveric elbow imaging on both scanners. Two radiologists rated CBCT scans to feature superior overall image quality compared to dose-equivalent MDCT studies. Reliability proved to be excellent between observers. This was further supported by the superior results of CBCT scans in objective image quality analysis of cancellous bone. The best distinction of the trabecula and bone marrow was achieved with the regular-dose scan protocol of the CBCT prototype. Despite four times lower radiation dose through tube current reduction, the computer-assisted evaluation of signal intensity distribution in the osseous tissue found no substantial difference between LD CBCT and RD MDCT studies. Observers even stated that LD CBCT studies featured better overall image quality (analysis of 


\section{Intermediate Signal Intensity Fractions}

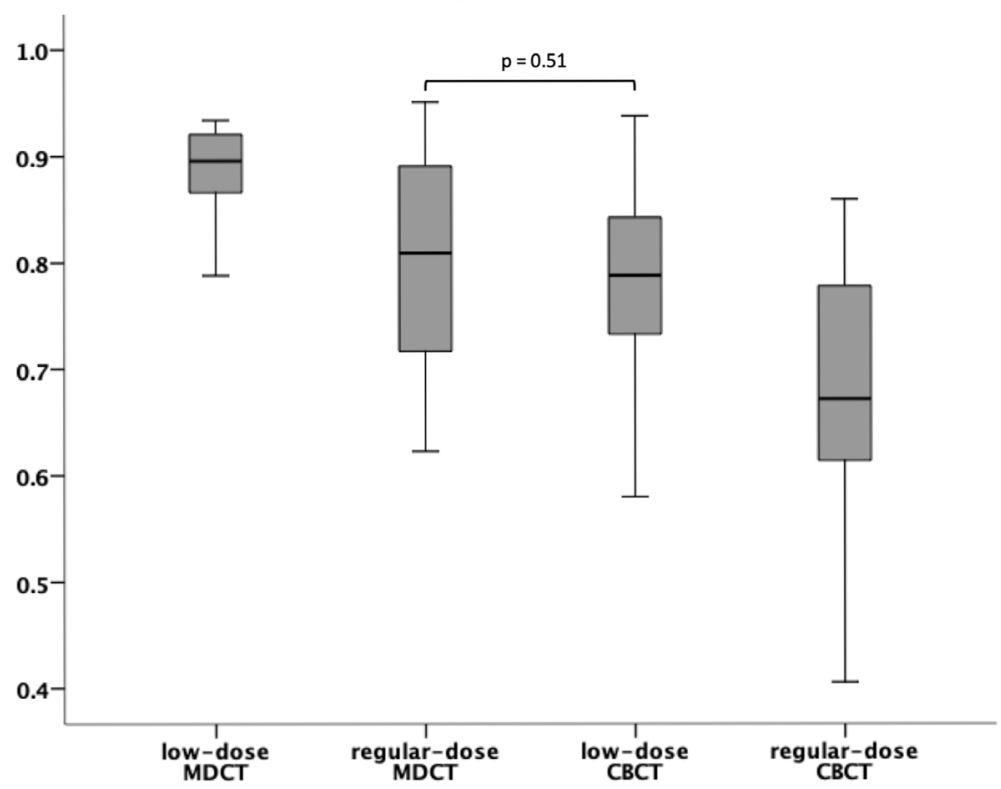

Fig. 4 Boxplots (median and 50\% of cases within the boxes) illustrate "undecided" pixel fractions within the intermediate range (25-75\%) of signal intensities for cone-beam computed tomography (CBCT) and multidetector computed tomography (MDCT) scans with dose-equivalent scan protocols. Smaller pixel fractions with intermediate signal intensity indicate superior image quality. Except for the difference between regular-dose MDCT and low-dose CBCT, all differences are statistically significant $(p<0.001)$
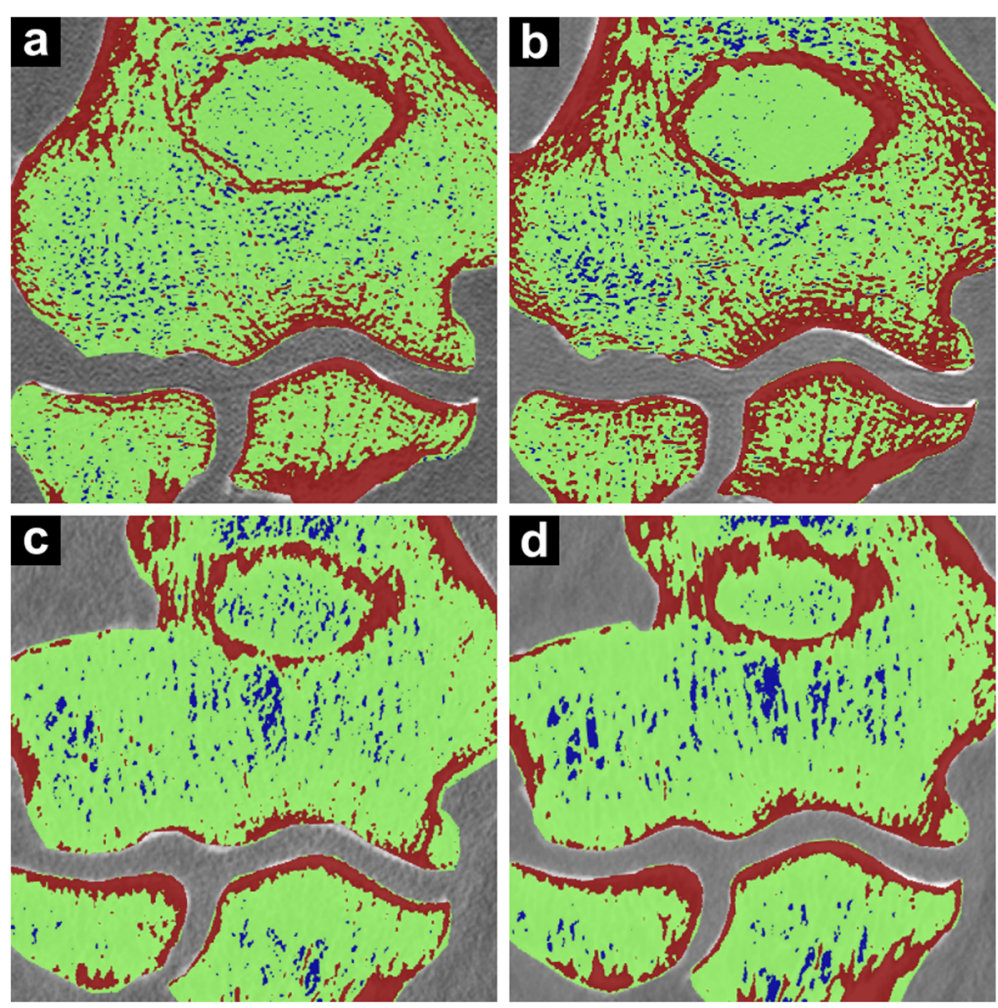

Fig. 5 Colour-coded visualisation of signal intensity distribution in cancellous bone for cone-beam computed tomography (CBCT) and multidetector computed tomography (MDCT) scans. Red regions contain the maximum quartile of grey values (75-100\%), while blue regions represent the minimum quartile $(0-25 \%)$. Green regions consist of pixels with intermediate signal intensity $(25-75 \%)$. a Low-dose $\mathrm{CBCT}$. b Regular-dose CBCT. c Low-dose MDCT. d Regular-dose MDCT 
bone and soft tissue) than RD MDCT studies. All LD CBCT studies were deemed diagnostic, whereas the application of the LD MDCT protocol resulted in approximately one third non-diagnostic images.

The superior image quality of the $\mathrm{CBCT}$ prototype can most likely be attributed to the twin robotic $\mathrm{x}$-ray system detector providing an isotropic voxel size of $149 \mu \mathrm{m}$, while the contemporary MDCT scanner has a pixel size of $300 \mu \mathrm{m}$ in the axial plane and $600 \mu \mathrm{m}$ in the $z$-direction. Furthermore, the limiting effect of the focal spot size on spatial resolution is reduced in CBCT scans by using a low-magnification acquisition geometry. In contrast to a previous ankle study with the commercially available CBCT scan mode, the tested prototype did not display more artefacts in bone than MDCT [15]. However, consistent with the literature [18], typical conebeam artefacts were still present in the soft tissue. While cone-beam scans were at least equal to MDCT concerning soft tissue noise, image noise in the bone was less pronounced for CBCT. The excellent depiction of bone microarchitecture and minimised radiation dose in CBCT scans has been one of the main reasons for its prominent role in oral and maxillo-facial imaging [19, 20]. However, unlike other CBCT scanners, the prototype 3D scan mode of the multi-use $\mathrm{x}$-ray system does not suffer from below-average image quality in the soft tissue [21]. Although trauma imaging of the elbow joint is primarily focused on bone display for fracture detection, the higher soft-tissue contrast can also be important, for example in occult fractures with concomitant effusion.

Regarding positioning options, the two telescopic arms and tableside scan trajectory of the twin robotic $\mathrm{x}$-ray system enable elbow scans in a comfortable supine or seated position with the upper extremity abducted by $90^{\circ}$. As patients with elbow trauma are frequently unable to adopt or maintain the challenging scan position necessary for optimal multidetector CT elbow studies, they may still be examined using the CBCT scan mode without higher radiation dose or loss of image quality.

Furthermore, the availability of radiography, fluoroscopy and CBCT imaging within the $\mathrm{x}$-ray suite allows for potential one-stop-shop imaging after trauma without additional patient transport or repeated positioning. Taking into account that elbow injuries (such as supracondylar humerus fracture) oftentimes affect children, the combination of first-rate 3D image quality and low radiation dose might particularly find application in paediatric radiology.

Limiting this study, 16 elbow joints from eight body donors were evaluated. As scans were exclusively performed on cadaveric specimens, possible motion artefacts cannot be ruled out for patient studies [3, 22]. The tested software prototype already reduced total scan time from 20 to $12 \mathrm{~s}$ compared to the current commercially available scan mode. However, this might still result in more artefacts through movement in clinical studies. The first tests of a marker-free auto-focus method based on the grey-level histogram entropy suggest that the acquisition time of the CBCT mode could be reduced even further by compensating deviations from the assumed scanning trajectory [23]. This approach should be evaluated in subsequent patient studies. As the tested CBCT prototype does not feature the same calibration for Hounsfield units as the MDCT scanner, signal-to-noise and contrast-to-noise ratios could not be compared in this study, subsequently limiting the availability of quantitative data. Furthermore, the impact of metallic implants or a cast around the elbow could not be analysed, because no osteosynthesis material was present in the field of view for any specimen. Formalin fixation is known to affect biological tissue samples over time, e.g., by breaking of hydrogen bonds and protein denaturation [24]. With specimens embalmed in formalin for several years before this study, optical density might have decreased, irrespective of imaging modality [25]. While Seidel et al. [26] found no substantial difference between the diagnostic accuracy of MDCT and CBCT scans of a cadaveric specimen with and without formalin fixation, particularly the long-term effects of such fixation are not fully understood. Moreover, despite the blinded evaluation of scans, readers might have become accustomed to the characteristic image traits of each scanner over the time of their reads.

Finally, we note that the presented CBCT prototype is currently not commercially available. Siemens Healthineers Multitom Rax is not available in all countries. Due to regulatory reasons, its future availability cannot be guaranteed.

In conclusion, the tested CBCT scan mode of the twin robotic $\mathrm{x}$-ray system provided better image quality than high-resolution MDCT for regular and low-dose scans of cadaveric elbows. The dedicated low-dose CBCT scan protocol delivered equally good distinction of bone microarchitecture and marrow as regular clinical MDCT studies despite one fourth of radiation dose, thus offering the potential for dose reduction.

\section{Abbreviations \\ 3D: Three-dimensional; CBCT: Cone-beam computed tomography; \\ $\mathrm{CTDI}_{\text {vol }(16 \mathrm{~cm})}$ : Volume computed tomography dose index (for 16-cm diameter polymethyl methacrylate dosimetry phantom); DLP: Dose-length product; ICC: Intraclass correlation coefficient; LD: Low-dose scan protocol $\left(C T D I_{\text {vol }(16 \mathrm{~cm})}=3.3 \mathrm{mGy}\right)$; MPR: Multiplanar reconstruction; R1: Reader 1; R2: Reader2; RD: Regular dose (clinical) scan protocol $\left(\mathrm{CTDI}_{\text {vol }(16 \mathrm{~cm})}=13.8 \mathrm{mGy}\right)$}

\section{Authors' contributions}

JPG analysed and interpreted all data. He also prepared the manuscript. AMW performed the statistical analysis and software-based estimation of the signal intensity distribution. ASK supported the draft of the manuscript and revised it for language. MWW and SE were involved in the scan preparation, 
supported data acquisition and revised the manuscript. CHG and LP also contributed to the acquisition of data and provided quality control. Observer analysis was performed by TG and SH. TG was also a major contributor to the preparation of the manuscript. TAB and RS designed and supervised the study. The authors read and approved the final manuscript.

\section{Funding information}

JPG was funded by the Interdisciplinary Center of Clinical Research Würzburg, Germany [grant number Z-2/CSP-06]. This publication was further supported by the Open Access Publication Fund of the University of Würzburg.

\section{Availability of data and materials}

The datasets used and/or analysed during the current study are available from the corresponding author on reasonable request.

\section{Ethics approval and consent to participate}

As body donors had voluntarily donated their corpses to the anatomical institute of our university for study and scientific purposes, separate institutional review board approval and written informed consent were not required. This work was carried out in accordance with the ethical standards of the institutional and national research committee and with the 1975 Declaration of Helsinki.

\section{Consent for publication}

Not applicable.

\section{Competing interests}

This study received a financial research grant by Siemens Healthineers (Erlangen, Germany). The authors of this manuscript declare no further relationships with any companies, whose products or services may be related to the subject matter of the article.

\section{Author details}

${ }^{1}$ Department of Diagnostic and Interventional Radiology, University Hospita Würzburg, Oberdürrbacher Straße 6, 97080 Würzburg, Germany. ${ }^{2}$ Institute of Anatomy and Cell Biology, University of Würzburg, Koellikerstraße 6, 97070 Würzburg, Germany. ${ }^{3}$ Institute for Diagnostic and Interventional Radiology, Faculty of Medicine and University Hospital Cologne, University of Cologne, Kerpener Straße 62, 50937 Cologne, Germany.

Received: 8 April 2020 Accepted: 3 July 2020

Published online: 08 September 2020

\section{References}

1. Miracle AC, Mukherji SK (2009) Conebeam CT of the head and neck, part 2: clinical applications. AJNR Am J Neuroradiol 30:1285-1292. https://doi.org/ 10.3174/ajnr.A1654

2. Koong B (2010) Cone beam imaging: is this the ultimate imaging modality? Clin Oral Implants Res 21:1201-1208. https://doi.org/10.1111/j.1600-0501. 2010.01996.x

3. Carrino JA, Al Muhit A, Zbijewski W et al (2014) Dedicated cone-beam CT system for extremity imaging. Radiology 270:816-824. https://doi.org/10. 1148/radiol.13130225

4. Konda SR, Goch AM, Leucht P et al (2016) The use of ultra-low-dose CT scans for the evaluation of limb fractures. Bone Joint J 98-B:1668-1673. https://doi.org/10.1302/0301-620X.98B12.BJJ-2016-0336.R1

5. Lintz F, Cesar Netto CD, Barg A, Burssens A, Richter M, Weight Bearing CT International Study Group (2018) Weight-bearing cone beam CT scans in the foot and ankle. EFORT Open Rev 3:278-286. https://doi.org/10.1302/ 2058-5241.3.170066

6. Tuominen EKJ, Kankare J, Koskinen SK, Mattila KT (2013) Weight-bearing CT imaging of the lower extremity. AJR Am J Roentgenol 200:146-148. https:// doi.org/10.2214/AJR.12.848

7. Benz RM, Hirschmann A (2016) 3D imaging of joints in the upright weightbearing position using multitom rax. J Trauma Treat 5:291. https://doi.org/ 10.4172/2167-1222.1000291

8. De Smet E, De Praeter G, Verstraete KLA, Wouters K, De Beuckeleer L, Vanhoenacker FMHM (2015) Direct comparison of conventional radiography and cone-beam CT in small bone and joint trauma. Skeletal Radiol 44:11111117. https://doi.org/10.1007/s00256-015-2127-3
9. Faccioli N, Foti G, Barillari M, Atzei A, Mucelli RP (2010) Finger fractures imaging: accuracy of cone-beam computed tomography and multislice computed tomography. Skeletal Radiol 39:1087-1095. https://doi.org/10. 1007/s00256-010-0911-7

10. Neubauer J, Benndorf M, Reidelbach C et al (2016) Comparison of diagnostic accuracy of radiation dose-equivalent radiography, multidetector computed tomography and cone beam computed tomography for fractures of adult cadaveric wrists. PLoS One 11:e0164859. https://doi.org/10. 1371/journal.pone.0164859

11. Subasi M, Isik M, Bulut M, Cebesoy O, Uludag A, Karakurt L (2015) Clinical and functional outcomes and treatment options for paediatric elbow dislocations: experiences of three trauma centres. Injury 46:S14-S18. https://doi.org/10.1016/j.injury.2015.05.026

12. Sheehan SE, Dyer GS, Sodickson AD, Patel Kl, Khurana B (2013) Traumatic elbow injuries: what the orthopedic surgeon wants to know. Radiographics 33:869-888. https://doi.org/10.1148/rg.333125176

13. Suresh P, Ninan T (2013) Computed tomography of hand and wrist. In: Davies AM, Grainger AJ, James SJ (eds) Imaging of the hand and wrist techniques and applications. Springer Berlin Heidelberg, pp 23-36. https://doi.org/10.1007/978-3-642-11146-4

14. Posadzy M, Desimpel J, Vanhoenacker F (2018) Cone beam CT of the musculoskeletal system: clinical applications. Insights Imaging 9:35-45. https://doi.org/10.1007/s13244-017-0582-1

15. Grunz J-P, Kunz AS, Gietzen CH et al (2019) 3D cone-beam CT of the ankle using a novel twin robotic X-ray system: assessment of image quality and radiation dose. Eur J Radiol 119:108659. https://doi.org/10.1016/j.ejrad.2019. 108659

16. Grunz J-P, Gietzen CH, Kunz AS et al (2020) Twin robotic X-ray system for 3D cone-beam CT of the wrist: an evaluation of image quality and radiation dose. AJR Am J Roentgenol 214:422-427. https://doi.org/10.2214/AJR.19.21911

17. Koo TK, Li MY (2016) A guideline of selecting and reporting intraclass correlation coefficients for reliability research. J Chiropr Med 15:155-163. https://doi.org/10.1016/j.jcm.2016.02.012

18. Schulze R, Heil U, Groß D et al (2011) Artefacts in CBCT: a review. Dentomaxillofac Radiol 40:265-273. https://doi.org/10.1259/dmfr/30642039

19. Roberts JA, Drage NA, Davies J, Thomas DW (2009) Effective dose from cone beam CT examinations in dentistry. Br J Radiol 82:35-40. https://doi. org/10.1259/bjr/31419627

20. Veldhoen S, Schöllchen M, Hanken H et al (2017) Performance of conebeam computed tomography and multidetector computed tomography in diagnostic imaging of the midface: a comparative study on phantom and cadaver head scans. Eur Radiol 27:790-800. https://doi.org/10.1007/s00330016-4387-2

21. Demehri S, Muhit A, Zbijewski W et al (2015) Assessment of image quality in soft tissue and bone visualization tasks for a dedicated extremity conebeam CT system. Eur Radiol 25:1742-1751. https://doi.org/10.1007/s00330014-3546-6

22. Nardi C, Molteni $\mathrm{R}$, Lorini $\mathrm{C}$ et al (2016) Motion artefacts in cone beam CT: an in vitro study about the effects on the images. $\mathrm{Br} J$ Radiol 89:20150687. https://doi.org/10.1259/bjr.20150687

23. Herbst M, Luckner C, Wicklein J et al (2019) Misalignment compensation for ultra-high-resolution and fast CBCT acquisitions. SPIE Proc 10948:57. https://doi.org/10.1117/12.2513276

24. Burkhart KJ, Nowak TE, Blum J et al (2010) Influence of formalin fixation on the biomechanical properties of human diaphyseal bone. Biomed Tech (Berl) 55:361-365. https://doi.org/10.1515/bmt.2010.043

25. Fonseca AA, Cherubini K, Veeck EB, Ladeira RS, Carapeto LP (2008) Effect of $10 \%$ formalin on radiographic optical density of bone specimens. Dentomaxillofac Radiol 37:137-141. https://doi.org/10.1259/dmfr/18109064

26. Seidel A, Bergauer B, Lell $M$ et al (2018) Does formalin fixation influence MSCT/CBCT accuracy? Surg Radiol Anat 40:31-37. https://doi.org/10.1007/ s00276-017-1908-x

\section{Publisher's Note}

Springer Nature remains neutral with regard to jurisdictional claims in published maps and institutional affiliations. 